Carmen Alberdi Urquizu

Universidad de Granada

\title{
LEPÉNISME ET MARINISME : VARIATIONS ET INVARIANTS DU DISCOURS POPULISTE IDENTITAIRE
}

\section{Lepenism and Marinism: Variations and Invariants of Populist Identity Discourse}

\begin{abstract}
The « Lepenism », the ideological-discursive system of the French Front national, seems to have generated in recent years a softened version of itself, the so-called « Marinism », aimed to ensure the normalisation of the party and the support of voters from other camps. However, the same nationalist and identity populism emerges underneath this policed surface, namely throughout the manipulation of the unspoken meanings.
\end{abstract}

Keywords: Lepenism, marinism, identity populism, de-demonisation, populist pathos

\section{Résumé}

Le « lepénisme », système idéologico-discursif du Front national, semble avoir engendré ces dernières années une version adoucie de lui-même - le « marinisme » - visant à assurer la normalisation du parti et l'adhésion d'électeurs venus d'autres camps. Or, sous la surface policée, transparaît la permanence des mythèmes et idéologèmes d'un même populisme nationaliste et identitaire, le non-dit dessinant, en filigrane, un parcours de retour à l'invariant.

Mots-clés : lepénisme, marinisme, populisme identitaire, dédiabolisation, pathos populiste

\section{Introduction}

Le populisme du Front national (FN) constitue, d'après Taguieff (2007), un hybride des versants protestataire, consistant dans la lutte contre les élites, et nationaliste-identitaire, basé, lui, sur l'idée d'une menace planant 
sur une identité culturelle et une nation conçue comme « homeland $»^{1}$. La nation s'y confond avec le peuple, une entité supposée homogène et monolithique, comprise dans la double acception du dêmos - le «petit peuple » qui se bat contre les « gros »- et de l'ethnos - le peuple comme nation à soubassement historico-ethnique. Or ce double versant penche nettement du côté de l'identitaire et subordonne le positionnement anti-establishment à la défense de l'identité nationale contre le complot immigrationniste ourdi par les élites mondialistes.

Le leader populiste, inscrit dans un scénario apocalyptique et manichéen, se représente lui-même comme une émanation de ce peuple, en lutte permanente contre un ennemi ramené à l'archétype essentialiste de l'immigré, incarnation du bouc émissaire. Fondé sur la polarisation exacerbée d'une auto-stéréotypie positive contre une hétéro-stéréotypie négative, son discours nourrit la haine de l'Autre et justifie l'exclusion. Ce nouveau racisme qui ne dit pas son nom n'a plus besoin de se fonder sur les vieux arguments biologico-raciaux : l'altérophobie trouve toute sa raison d'être dans l'inassimilabilité de ce qui est différent. Une rhétorique efficace, axée sur la peur, est mise au service de cette stratégie de «victimperpetrator reversal» (Wodak, 2015). Du lepénisme au marinisme, elle tend néanmoins à se dissimiler sous une évolution formelle apparente qui se veut l'emblème d'une normalisation idéologique. Nous en aborderons les variations et les invariants à partir d'un corpus de discours de Marine Le Pen prononcés en 2017 lors de la campagne des présidentielles, regroupant le discours d'ouverture de campagne à Fréjus (17.09.16), le premier meeting de l'entre-deux-tours à Nice (27.04.17) et le meeting de clôture à Villepinte (01.05.17). Ces trois discours semblent particulièrement représentatifs de la malléabilité du discours populiste dans la mesure où ils sont censés cibler des audiences de plus en plus larges, du FN au camp mélenchonien, puis républicain.

\section{La dédiabolisation : variations de surface}

Comme la plupart des partis classés à l'extrême-droite, le FN a entrepris une normalisation, processus que Wodak (2015) nomme " haiderization », baptisé pour le FN sous le terme « dédiabolisation ». Il s'agirait, d'après ce que Marine Le Pen rapporte dans son autobiographie (2006), d'une mission qu'elle se serait donnée en 2002, au lendemain de l'échec de

\footnotetext{
${ }^{1}$ Le slogan le plus prisé dans les meetings frontistes est bien « On est chez nous! ».
} 
Jean-Marie Le Pen au second tour des présidentielles. Accompagnée de Louis Aliot, " artisan influent » de la dédiabolisation (Rosso, 2011), elle assume depuis lors la présidence de Générations Le Pen, organe de renouveau générationnel devant permettre de " déracialiser » le parti (Lebourg, 2015). En 2009, le projet bénéficie d'un apport significatif du point de vue programmatique avec la collaboration de Florian Philippot, responsable d'un virage républicain et étatique. Après l'accès de Marine Le Pen à la présidence du parti en 2011, le processus se poursuit de plus belle et semble pleinement aboutir en 2015, année de la rupture avec JeanMarie Le Pen - à la suite d'un énième dérapage - sanctionnée par son expulsion du parti et le changement de nom du FN, devenu en avril 2018 le Rassemblement National (RN).

Divers signes de cette « dé-filiation » sont affichés ostensiblement sur les supports de campagne en 2017 : suppression du nom du parti et du nom de famille et remplacement de l'emblème historique du parti - la flamme tricolore - par une rose bleue. Or la normalisation se veut non seulement visible, mais surtout audible. Un nouvel ethos discursif la cristallise, censé véhiculer les nouvelles idées : le "marinisme », qui entend marquer ses distances vis-à-vis du lepénisme et de la ligne dure du parti.

L'objectif essentiel reste d'éviter tout dérapage xénophobe ou antisémite, mais aussi d'euphémiser toute tournure trop connotée, à l'instar de la " préférence nationale », devenue "priorité nationale ». Le concept, développé par Jean-Yves Le Gallou en 1985, présuppose le besoin de fournir aux "Français de souche », «blancs, de civilisation européenne et de religion ou d'héritage chrétien » (Albertini \& Doucet, 2016 : 200), les armes pour la reconquête intellectuelle, politique et morale de la France. Il devient la colonne vertébrale, l'ADN du FN et fait converger l'ensemble des mesures économiques et sociales (Dély, 2017a ; Lafarge, 2016) vers un objectif essentiel : réserver aux seuls Français tous les emplois, les logements, les aides sociales. La notion de " préférence ", à forte résonance identitaire, a cependant fait l'objet d'une substitution visant à l'euphémisme : à ce terme qui laisse entendre la subjectivité, l'on préfère désormais celui de " priorité » qui connote la rationalité, le choix basé sur l'efficacité managériale, la réponse rapide face à une situation d'urgence (Rosso, 2011 ; Alduy \& Wahnich, 2015 ; Eltchaninoff, 2017). L'antithèse récurrente dans les discours frontistes nouvelle génération : la "préférence étrangère », est sous-tendue par ce même concept, ainsi que le slogan, fréquent sur Twitter sous forme de hashtag, «Les nôtres avant les Autres!» 
Dans notre corpus, de Fréjus à Nice et à Villepinte, on assiste à un effacement quasi total des fondamentaux de la francité lepéniste - l'identité, la souveraineté - et à une diminution remarquable de l'emploi de mots à forte coloration frontiste - nation et peuple. De subtils glissements sémantiques permettent d'enrichir divers axiologèmes de nouvelles nuances - tel le mot-valeur patriotisme, désormais utilisé en collocation avec économique. Enfin, par appropriation et resémantisation des mots des adversaires politiques - véritable « OPA sémantique » (Fourest \& Venner, 2011 ; Alduy \& Wahnich, 2015) -, l'interdiscours crée un sous-texte adapté aux divers auditoires ${ }^{2}$ et livre diverses clés d'interprétation sur le principe de «l'ambivalence calculée » (Wodak, $2015: 53$ ).

La dédiabolisation s'appuie aussi sur une puissante campagne de médiatisation. Depuis son début télévisuel sur France 3 en 2002 pour commenter la défaite du FN, Marine Le Pen se révèle dotée d'un indiscutable charisme télégénique et devient vite ce que les médias considèrent être une « bonne cliente » (Rosso, 2011 ; Dézé, 2015). Ceci va de pair avec une certaine peopolisation (Fourest \& Venner, 2011) du personnage, dont la vie personnelle et sentimentale commence à occuper les pages des magazines. Le but est de construire un ethos de proximité ${ }^{3}$ sur l'image d'une femme moderne, conciliant féminité, maternité et grandes responsabilités politiques, et de contrecarrer la virulence tribunitienne du père. À cela contribuent également sa forte présence sur les réseaux sociaux et la construction d'une « $e$-réputation » fondée sur le nombre de ses followers ${ }^{4}$. Sur ce point, elle ne fait que suivre encore les pas de son père. En effet, le FN a été le premier parti en France à se doter, en 1996, d'un site web - conçu par Pierre Sautarel, créateur de Fdesouche, le site le plus visité de la fachosphère - et un des premiers à ouvrir un compte Facebook en 2006.

Depuis ses origines, Internet apparaît aux frontistes comme un média alternatif capable « d'inverser le rapport de force dans la guerre culturelle que se livrent 'mondialistes' et 'patriotes' - entre les médias du 'système' et les petits soldats de la 'réinformation' » (Albertini \& Doucet, 2016 :

2 Pour une analyse de l'emploi des axiologèmes et de l'interdiscours dans ce corpus, voir Alberdi (2018).

3 À cet égard, voir aussi Bonnafous (2003), Boudillon (2005), Dély (2017a).

${ }^{4}$ Il faut néanmoins rester prudent à l'égard de ces métriques du web, devenues des indices de « popularité » en temps réel : une étude aurait ainsi prouvé en 2014 que 85,5\% des fans de Marine Le Pen sur Twitter cachaient de faux comptes créés par des robots ou correspondaient à des comptes inactifs (Boyadjian, 2015). 
213). Rien d'étonnant dès lors à leur ferme opposition au projet de loi anti-fake news d'Emmanuel Macron. Le RN multiplie les déclarations et les communiqués de presse dénonçant cette loi « liberticide » (Marine Le Pen, Causeur, 04.07.18), qui illustre la « censure politique imposée par Bruxelles » (Dominique Bilde, 05.01.18) et constitue un «premier pas vers le Ministère de la Vérité d'Orwell » (Sébastien Chenu, 08.01.18).

Comme le fait remarquer Henry (2017), si Marine Le Pen «se laisse rarement aller aux mensonges éhontés, [...] elle sait en revanche prendre ses distances avec la vérité », comme l'ont mis en évidence pendant la campagne présidentielle et lors du débat de l'entre-deux-tours les rubriques de presse dédiées au fact-checking - "Les Décodeurs » pour Le Monde et « Desintox » pour Libération. Elle étaye souvent ses arguments par des nouvelles peu ou pas vérifiées, tirées notamment de Fdesouche, et n'hésite pas à les rediffuser sur son compte Twitter. Elle parvient, ce faisant, à entretenir un double discours ${ }^{5}$ : d'une part, une " communication numérique top-down, fortement contrôlée et centralisée » des contenus du parti, reproduits sans variation sur les divers comptes officiels (Boyadjian, 2015) ; de l'autre, une promotion subliminale de la post-vérité qui passe par le partage de fake news et de faits alternatifs tirés de la fachosphère.

Protégée par l'anonymat des pseudonymes et la prétendue liberté d'expression, la parole numérique se prête aisément au dérapage, d'autant plus que l'absence d'investissement énonciatif du retweetage forme de discours rapporté, relevant de la catégorie des actes délocutifs (Charaudeau, 1992) - favorise ce que Wodak (2015 : 19-20) nomme le « right-wing perpetuum mobile»: provoquer délibérément le scandale, nier l'intention attribuée à l'auteur, recadrer et redéfinir l'objet du sandale pour se déclarer enfin victime d'une persécution. Cette stratégie aboutit souvent à un simulacre d'excuse mettant l'accent non pas sur le contenu posé mais sur la mauvaise interprétation qui en a été faite, ce par quoi le pseudo-énonciateur laisse entendre à ses inconditionnels qu'il demeure bien celui qui ose dire tout haut ce que les gens pensent tout bas (L'Heuillet, 2017), mais aussi celui qui sait faire passer le dire sous les espèces du non-dit.

\footnotetext{
5 Voire un discours dédoublé, Marine Le Pen n'étant que le pendant dédiabolisé de la vraie Marine qui, elle, s'exprimerait sans gêne et sans contrainte sur son autre compte Twitter sous le pseudonyme d'Anne Lalane, comme l'ont dévoilé divers médias les 16 et 17 juin 2017.
} 


\section{Le non-dit : l'invariant des mythèmes frontistes}

Le contenu passé sous silence, qu'il relève de l'incapacité ou du refus de dire, constitue toujours un " acte énonciatif in absentia " (Van den Heuvel, 1985 : 67) ouvrant sur des virtualités de sens que seul le contexte permet de réduire. Le marinisme, instaurant sans cesse un jeu entre posé et présupposé et bâti sur des ambivalences calculées, constitue en fait un langage codé, susceptible de dérouter les néophytes mais adressant encore suffisamment de signes de connivence aux initiés, signes d'autant plus précieux qu'ils se font rares alors même qu'ils renforcent le sentiment d'appartenance. C'est notamment à Fréjus que cette connivence se fait sentir : en effet, pris dans une double contrainte, adressé en première instance à la base frontiste mais voué en même temps à une large diffusion ${ }^{6}$, le discours doit garder sa surface policée tout en essayant d'atteindre par ricochet le vieux Front.

Le non-dit relevant de l'incapacité procède des tabous liés à la dédiabolisation, parmi lesquels figure désormais toute référence au père évincé, «mort » de ses propres excès. Mais l'indicible revient sous forme d'allusions plus ou moins voilées dans le discours de la « candidate du peuple », prête comme son père à redonner à la France sa grandeur. Il en est ainsi, par exemple, de l'appel aux Français, « quelles que soient leurs origines, opinions, parcours » (Fréjus), qui n'est pas sans rappeler l'allocution de son père après le premier tour de la campagne présidentielle en 2002, que Marine Le Pen décrit elle-même dans son autobiographie (2006 : 223) comme « une de ses plus belles interventions sur la cohésion nationale », et qui aurait été écrite par Louis Aliot (Rosso, 2011). Également adressée à ceux qui savent lire entre les lignes, l'allusion au père clairvoyant injustement caricaturé, prophète du monde qui vient. La prescience, qui va de pair « avec le trope millénariste d'une catastrophe dont l'imminence requiert la mobilisation salutaire » (Alduy \& Wahnich, 2015 : 161), constitue le don du Sauveur, figure clé, avec le mythe de la décadence et la dénonciation de la source du mal, de la dramaturgie classique du pathos populiste (Charaudeau, 2008).

Le motif de la décadence, fin d'un Âge d'Or à reconquérir, est omniprésent dans le discours de Fréjus et appelle en surface le nom le plus

6 Tous les discours sont diffusés sur le site web de campagne (https://www.marine2017. fr), lancé le 14 juillet 2016 et actuellement indisponible, mais aussi sur Youtube et relayés par les comptes officiels Facebook et Twitter de Marine Le Pen, du parti et des cadres frontistes. 
fréquemment répété dans le corpus : France, une France dépossédée dont le déclin est décrit dans ses moindres détails. Tout en signalant par antiphrase l'inutilité « de multiplier les exemples de situations injustifiables » vécues « tous les jours », Marine Le Pen énumère toutes les victimes du contrôle étranger et dépeint une situation apocalyptique, offrant à tout un chacun la possibilité de s'y reconnaître. Cette menace, palpable et quotidienne, éveille l'angoisse, renforcée par l'imminence d'une destruction avançant à grands pas :

Chaque année, s'allonge la longue liste des entreprises [...] sous le contrôle de capitaux étrangers [...]. Chaque semaine, des salariés découvrent [...] que leur entreprise a quitté le territoire. Chaque jour [...] nous sommes invités à troquer [...] les illusions de la démocratie, de la souveraineté et de la Nation, contre les petits profits de la soumission (Fréjus).

Elle suscite encore l'inquiétude à grand renfort de chiffres suivant une croissance exponentielle : «Ce bilan se mesure aussi en dizaines de milliers d'entreprises détruites, en millions d'emplois perdus, en milliards d'aides publiques détournées ».

La source du mal, le complot pour l'islamisation, le Grand Remplacement ${ }^{7}$ et la destruction de la France dont le migrant est l'instrument, est progressivement dévoilée dans les discours successifs, d'abord à Nice ${ }^{8}$, " en décryptant le Macron dans le texte », puis enfin à Villepinte, où « le masque tombe ». Considéré sous divers éclairages, Macron apparaît comme un pantin dont les ficelles sont tirées par un double totalitarisme - mondialisation et islamisme, le «tout-commerce » et le «tout-religion». Le retour sur l'invariant est implicitement opéré à travers une collocation devenue récurrente dans le marinisme : le banquier d'affaires, un cliché qui renouerait avec le vieux discours antisémite et par-là, avec la vieille garde frontiste (Dély, 2017b). Marine Le Pen semble ainsi jouer sur un « antisémitisme subliminal », sorte de piqûre de rappel pour les secteurs les plus récalcitrants (Eltchaninoff, 2017 : 136), fondé non plus sur le

7 La théorie du Grand Remplacement, popularisée par Renaud Camus, n'est pas nouvelle : elle puise ses sources, entre autres, dans les visions cauchemardesques de $L a$ France juive d'Édouard Drumont (1886), à cette différence près que l'islamophobie s'est substituée à l'antisémitisme dans le marinisme (Kauffmann, 2016).

8 On constate en effet l'absence à Fréjus de toute référence au terrorisme et à l'islamisme, malgré la proximité temporelle de l'attentat de Nice, survenu deux mois auparavant. Il en va tout autrement à Nice où sont mobilisés les termes de terroriste/me (10 occurrences, 5 à Villepinte), islamiste/me (12 et 3), Frères musulmans (1 et 1), UOIF (1 et 1), attentat (2), salafisme (1) et charia (1). 
cliché du peuple déicide ou les arguments du racisme social, mais sur le stéréotype du banquier, des élites « cosmopolites » et " nomades », et de l'idolâtrie de l'argent incarnée dans les figures, récurrentes, de Jacques Attali, Goldman Sachs ou la banque Rothschild.

Le deuxième cas de figure, le refus de nommer, revient à nier l'existence. Tel est le cas d'un terme élu mot de l'année 2016 en France et totalement absent du corpus : réfugiés. Le besoin de distinguer entre migrants et réfugiés, débat qui a largement occupé les médias et les discours politiques au cours de l'été 2015, n'est pas fondé pour Marine Le Pen :

Je pense que les réfugiés politiques sont ultra-minoritaires. [...] Moi, j'ai vu les images des clandestins qui descendaient, qui étaient emmenés en Allemagne de la Hongrie, etc. Eh bien, dans ces images, il y a 99 \% d'hommes. Or, moi, je pense que des hommes qui quittent leur pays pour laisser leur famille làbas, ça n'est pas pour fuir la persécution. C'est évidemment pour des raisons économiques (Le Point, 08.09.2015).

Nul besoin dès lors de nommer une réalité inexistante que les médias et la bien-pensance gauchiste manipulent pour cacher un problème purement économique, coupé de toute dimension humaine. La déshumanisation et l'abstraction constituent des éléments clé de cette rhétorique antiimmigration fondée sur la peur. Le discours recrée la menace, anxiogène et protéiforme, d'une invasion par une masse de " clandestins » - criminalisés même avant leur arrivée -, amplifiée dans l'imaginaire social par un discours médiatique la présentant toujours sous la forme de « vagues », de «flux incessants », de « débarquements » (Orrù, 2017). " Incontrôlée », l'immigration est toujours « massive », redoutable dans la mesure où elle vise à la « submersion », mais aussi abstraite, le discours ne mettant jamais l'accent sur la figure des immigrés mais sur le «solde migratoire », ce qui constitue d'ailleurs un rempart contre les accusations de racisme (Alduy \& Wahnich, $2015: 75$ ).

L'anti-immigration se dilue donc dans un récit qui la rationalise et la prive de toute dimension passionnelle. Étayé à grand renfort de chiffres et de statistiques, le lien de causalité entre immigration et menace contre la stabilité économique justifie des mesures radicales découlant tout naturellement du «bon sens ». Ce volet économique se décline différemment selon qu'il doit convaincre les segments les plus favorisés, défenseurs du « chauvinisme du bien-être » (Taguieff, 2012), ou les plus précarisés, sujets à la peur du chômage et de l'instabilité. En milieu 
frontiste, comme à Fréjus, ce populisme protestataire cède la place aux nuances identitaires.

En adaptant ses arguments aux circonstances et aux publics ciblés, Marine Le Pen fait ainsi varier la forme du discours sans remettre en question son socle fondamental et continue d'accorder la place essentielle à la lutte anti-immigration. Elle répond ainsi parfaitement aux peurs et angoisses d'une société fragilisée par le processus de mondialisation (Dominici \& Alessandri, 2017 : 125).

\section{Conclusion}

Malgré les essais de normalisation et les manipulations sémanticodiscursives, l'anti-immigration reste bien la clef de voûte du RN. Candidate du peuple au charisme télégénique et numérique, voulant rassembler autour d'elle la totalité de la nation-peuple dans la défense d'une identité commune contre les «élites illégitimes » et les " étrangers indésirables » qu'elle a seule le courage de combattre, Marine Le Pen reste donc foncièrement attachée au même socle idéologique et discursif que JeanMarie Le Pen : celui du national-populisme à inspiration identitaire. L'exacerbation de ce dernier trait induit " une dérive vers une forme de racisme plus différentialiste-culturel qu'inégalitariste-biologisant » (Taguieff, 2012 : 62). Un « racisme élégant » (González Alcantud, 2011) ou « racisme apophatique » (Dominici \& Alessandri, 2017), apparemment invisible et visiblement banalisé par l'action conjointe des médias et des réseaux sociaux numériques, qui imprègne subrepticement l'imaginaire social et les usages discursifs.

\section{Bibliographie}

Alberdi, C. (2018). Le «marinisme». Axiologèmes et interdiscours au service de la polysémie. In Pamies, A., Balsas, I. M. \& Magdalena, A. (ed.), Lenguaje figurado y competencia interlingüística (I). Aspectos teóricos, pp. 141-150. Granada: Comares.

Albertini, D. \& Doucet, D. (2016). La Fachosphère. Comment l'extrême droite remporte la bataille d'Internet. Paris: Flammarion.

Alduy, C. \& Wahnich, S. (2015). Marine Le Pen prise aux mots. Paris: Seuil.

Bonnafous, S. (2003). Femme politique: une question de genre? Réseaux, 120, pp. $119-145$.

Boudillon, J. (2005). Une femme d'extrême droite dans les médias. Le cas de Marine Le Pen. Mots, 78, pp. 79-89. 
Boyadjian, J. (2015). Les usages frontistes du web. In Crépon, S., Dézé, A. \& Mayer, N. (ed.), Les faux-semblants du Front national: sociologie d'un parti politique, pp. 141-160. Paris: Presses de Sciences Po.

Charaudeau, P. (1992). Grammaire du sens et de l'expression. Paris: Hachette.

Charaudeau, P. (2008). Pathos et discours politique. In Rinn, M. (ed.), Émotions et discours. L'usage des passions dans la langue, pp. 49-58. Rennes: P.U. de Rennes.

De Calan, M. (2016). La vérité sur le programme du Front national. Paris: Plon.

Dély, R. (2017a). La vraie Marine Le Pen. Une bobo chez les fachos. Paris: Plon.

Dély, R. (2017b). Le «banquier d'affaires insensible», ce vieux cliché antisémite que Marine Le Pen recycle pour Macron. Marianne, 28.04.17.

Dézé, A. (2015). La construction médiatique de la «nouveauté» FN. In Crépon, S., Dézé, A. \& Mayer, N. (ed.), Les faux-semblants du Front national: sociologie d'un parti politique, pp. 453-504. Paris: Presses de Sciences Po.

Dominici, T. \& Alessandri, J.-L. (2017). The Front National's Populism: From the Far Right to the Normalization of an Identity Parti. In Kudors, A. \& Pabriks, A. (ed.), The Rise of Populism: Lessons for the European Union and the United States of America, pp. 119-132. Riga: University of Latvia Press.

Eltchaninoff, M. (2017). Dans la tête de Marine Le Pen. Arles: Actes Sud.

Fourest, C. \& Venner, F. (2011). Marine Le Pen démasquée. Paris: Grasset.

González Alcantud, J. A. (2011). Racismo elegante. De la teoría de las razas culturales a la invisibilidad del racismo cotidiano. Barcelona: Bellaterra.

Kauffmann, G. (2016). Le nouveau FN. Les vieux habits de l'extrême droite. Paris: Seuil.

Lafarge, S. (2016). Front national et la France catho-facho-complo. Createspace Independent Publishing Platform.

Le Pen, M. (2006). A contreflots. Paris: Grancher.

Lebourg, N. (2015). Le Front national et la galaxie des extrêmes droites radicales. In Crépon, S., Dézé, A. \& Mayer, N. (ed.), Les faux-semblants du Front national: sociologie d'un parti politique, pp. 121-140. Paris: Presses de Sciences Po.

L'Heuillet, H. (2017). Tu haïras ton prochain comme toi-même. Penser la haine de notre temps. Paris: Albin Michel.

Orrù, P. (2017). Il discorso sulle migrazioni nell'Italia contemporanea. Un'analisi linguistico-discorsiva sulla stampa (2000-2010). Milan: Franco Angeli.

Rosso, R. (2011). La face cachée de Marine Le Pen. Paris: Flammarion.

Taguieff, P.-A. (2007). L'illusion populiste. Essai sur les démagogies de l'âge démocratique. Paris: Flammarion.

Taguieff, P.-A. (2012). Le nouveau national-populisme. Paris: CNRS.

Van den Heuvel, P. (1985). Parole, mot, silence. Pour une poétique de l'énonciation. Paris: José Corti.

Wodak, R. (2015). The Politics of Fear. What Right-Wing Populist Discourses Mean. Londres: Sage. 